\title{
Eliminating guidewire retention during ultrasound guided central venous catheter insertion via an educational program, a modified CVC set, and a drape with reminder stickers
}

Wee Ming Peh, Wann Jia Loh, ghee chee phua, Chian Min Loo

Singapore General Hospital, Singapore

\begin{abstract}
Guidewire retention is a severe but preventable complication from central venous catheter (CVC) insertion. There were three cases of guidewire retention during CVC insertion in the medical intensive care unit (MICU) in Singapore General Hospital, in the period between December 2011 and February 2012. The primary objective of this quality improvement project was to eliminate future incidences of guidewire retention during CVC insertion in the MICU and medical intermediate care area (MICA) via a structured educational program and a cost effective modified CVC set. The secondary objective was to perform a cost analysis and comparison between the use of the conventional hospital CVC set and drape with our newly modified CVC dressing kit.

Root cause analysis of the three cases identified major factors leading to guidewire retention. Interventions were planned and tested using PDSA cycles. Internal medicine trainees rotating through MICU and MICA during the period between February 2012 and June 2013 underwent a multi-modal structured CVC insertion training program with hands on simulation. They also used a newly modified CVC dressing kit and drape. The CVC dressing kit was modified (CVC PLUS) to include a sterile drape with reminder stickers stating "REMOVE the GUIDEWIRE," as well as a sterile ultrasound sleeve. The total number of CVC insertions performed and guidewire retentions were monitored.

During the period of study there were $320 \mathrm{CVC}$ insertions in the MICU and MICA. Since this quality improvement project was initiated, and up to the submission of this article, there have not been any further cases of guidewire retention in the MICU and MICA. The total cost reduction per use of CVC PLUS was $\$ \$ 29.26$ (Singaporean Dollars).
\end{abstract}

A multi-modal structured training program, integrated with a modified, pre-packed CVC set, and drapes with reminder stickers (all included in CVC PLUS) were cost effective, and improved patient safety by eliminating guidewire retention during CVC insertion.

\section{Problem}

There were three cases of guidewire retention out of 120 ultrasound guided central venous catheter (CVC) insertions in the medical intensive care unit (MICU) of Singapore General Hospital between December 2011 and February 2012. Guidewire retention during CVC insertion can be associated with significant morbidity to the patient. These alarming incidences necessitated urgent action to implement a cost effective intervention to eliminate guidewire retention during insertion of the CVC.

\section{Background}

More than five million CVCs are inserted into patients in the United States alone each year, for the purposes of monitoring central venous pressure, to administer fluids and medications, and to perform haemodialysis.[1] The insertion of a CVC can be associated with complications such as pneumothorax, haemothorax, fracture of guidewire, loss of guidewire, and retention of guidewire during the procedure. $[1,2,3,4,5,6,7,8,9]$

A retained guidewire may fracture, cause thromboembolic complications, become infected, and lead to cardiac perforation and tamponade.[10, 11, 12] The loss and retention of guide wires during CVC insertion procedures can be prevented.[10]

The primary objective of this intervention is to eliminate the future incidences of guidewire retention, via a structured multi-modal education program with CVC set modification, and a drape with reminder stickers.

As a secondary objective, we evaluated the cost drivers and overall unit cost of using the CVC PLUS and drape in a typical insertion of a CVC in the MICU and MICA, as compared to conventional, reusable, hospital assembled equipment.

\section{Baseline measurement}

Prior to the period between December 2011 and February 2012, there were no reported incidences of guidewire retention in the MICU and MICA at Singapore General Hospital for 10 years. However, the true incidence of guidewire loss after placement of CVC is unknown due to under-reporting. In another tertiary hospital, guidewire loss occurred at a rate of one per 3291 procedures.[6]

Prior to the initiation of this quality improvement project, medical 
BMJ Quality Improvement Reports

residents learnt ultrasound (US) guided CVC insertion via opportunistic bedside teaching. Residents were educated by a brief didactic lecture. They then observed and assisted seniors in performing this procedure on patients, and subsequently performed the procedure themselves. Prior to CVC insertion, a nurse would prepare a conventional toilet and suture set, four reusable sterile drapes, an additional reusable sterile drape for the ultrasound transducer, sterile gel, and a disposable scalpel. The procedurist would then set aside equipment not needed for the CVC insertion procedure. He would create a sterile field with the four sterile drapes, and would wrap the additional sterile drape around the transducer with assistance by the nurses. The preparation phase requires good organisation and cooperation by the nurses.

Although many became familiar with performing the procedure through experience, there was no organised teaching on the complications and risks involved in the procedure, nor an explanation of measures to reduce these risks.

To identify the contributing factors leading to the retention of the guidewire a multidisciplinary project team was formed, including internal medicine residents, medical intensivists, and specialized nurses from the MICU and MICA.

Personnel involved in the three guidewire retention incidents (supervising attending doctors, procedurists, and nurses) were interviewed by the multidisciplinary team. The interview attempted to identify the factors contributing to retention of the guidewire in each case.

A questionnaire survey was given to a group of medical officers and residents who had completed a critical care posting prior to the implementation of our quality improvement measures. Fifty percent rated their competency and knowledge of complications with CVC line insertion to be suboptimal.

Root cause analysis of the three cases and the survey identified major factors leading to such complications. Interventions were planned and tested using PDSA cycles. Root cause analysis revealed that the major factors leading to a retained guidewire during the CVC insertion procedure were the following:

Procedurist factors

1. Insufficient education and training: insufficient practice, lack of confidence, poor technique

2. Lack of awareness of CVC insertion complications among the residents

3. Insufficient supervision

4. Lack of reminders

Equipment factors

1. Non-user friendly CVC insertion set and drape

2. Cluttering of the procedure site with large amounts of equipment and distractions with unnecessary equipment

See supplementary file: ds6954.pdf - "Annex 1 final_2"

\section{Design}

The MICU and MICA were chosen as the implementation sites for our quality improvement project. All internal medicine residents who rotated through the MICU and MICA as part of their internal medicine critical care residency training, in the period between June 2012 and February 2013, took part in the structured training program and participated in the surveys.

Addressing the challenges identified from the root cause analysis, we instituted the following interventions via PDSA cycles:

1. A multi-modal structured training program, with knowledge acquisition and practical skills training

2. Modification of the CVC dressing set

3. Modification of the CVC drape to include reminder stickers to remove the guidewire

4. Compulsory supervision of CVC line insertion.

To evaluate the cost of the custom CVC set and drape as part of the intervention to reduce guidewire retention, we calculated the unit cost for each CVC procedure which was the sum of the following:

1. Net material cost for insertion (CVC set and drape)

2. Manpower cost ((cost/hr of procedurist $+\operatorname{cost} / \mathrm{hr}$ of ancillary staff) $x$ average time to complete the procedure)

3. Waste management cost (cost $/ \mathrm{kg}$ of waste $\mathrm{x}$ waste produced $/ \mathrm{kg}$ )

The Singapore General Hospital finance department and Central Sterile Supplies Department provided the information on cost of materials, manpower, and waste management involved in using the conventional reusable hospital assembled equipment and drape per insertion procedure. We also obtained the material, manpower, and waste management costs involved in the use of the modified CVC PLUS set and sterile drapes from the medical supply vendors, and compared the best prices.

\section{Strategy}

PDSA cycle 1

We planned to increase awareness of the complications of guidewire retention and increase residents' confidence in the CVC insertion technique. Thirty nine residents rotating through the MICU and MICA between June 2012 and February 2013 received a didactic lecture on the technical aspects of inserting a CVC at the beginning of the posting. The main issues discussed were landmark techniques, image analysis on the bedside ultrasound, complications associated with CVC insertion, and management of these complications. Awareness of guidewire removal was emphasized repeatedly during the training program.

Residents prepared agar filled with balloons pre-filled with dye and used them to perform transverse and longitudinal ultrasound localisation of "blood vessels," and to appreciate real time images of 
puncturing blood vessels (annex 1, figure 1).

Residents also practiced visualization of internal jugular veins on each other during the sessions, and simulated insertions of the CVC using a mannequin (annex 1, figure 2).

Most residents found the training program to be useful, and their confidence increased after completion. The procedural skills of the residents were assessed throughout the posting.

One of the important feedback points from the residents during the implementation of this intervention was the lack of reminders. The residents involved in the intervention found the original CVC set and sterile towels were not user friendly, and there were multiple unnecessary items in the original kit that were not useful. The previous practice of using sterile towels to wrap around the ultrasound probe was found to be tedious. In addition, supervision was not always provided, as supervisors may have been occupied with other duties during the procedure.

The feedback was similar to the points identified during the root cause analysis performed earlier. They were prioritized and incorporated into the next PDSA cycle.

PDSA cycle 2

We sought to incorporate reminder aids, improve the CVC set and drape user-friendliness, and aimed to increase supervision during CVC insertion.

The CVC set was modified to a custom made set which included only the necessary items required during CVC insertion. This was called CVC PLUS (annex 1, figure 4). Four sterile cloths for creating the sterile field around the cannulation site (annex 1, figure 5) were replaced with a single sterile drape, with reminder stickers to remove the guidewire (annex 1, figures 6 and 7). An instructional video was created and uploaded on the hospital website, to teach residents how to use the single drape with reminder stickers and the sterile ultrasound sleeve in a sterile and effective manner.

Lastly, our team sought the cooperation of the head of the respiratory and critical care medicine department to enforce that all CVC lines must be supervised diligently by registrars or consultants at all times.

The structured training program with CVC PLUS was integrated into the CVC training program for residents in the MICU and MICA between the period of June 2012 to February 2013.

To evaluate the effect of the intervention, the number of CVC insertions were monitored by the nurses and doctors of the MICU and MICA during the intervention period. Any cases of guidewire retention were reported to project group members by the MICA and MICA nurses and doctors. A survey was performed at the beginning and end of the posting.

\section{Results}

Between June 2012 and February 2013 there were 320 CVC insertions in the MICU and MICA. There were no incidences of guidewire retention during CVC insertion following the implementation of the quality improvement measures, and this remains true at the point of submission of this article (Feb 2016).

Ninety seven percent (38/39) of participants found the educational training program to be very useful and informative. The two areas of excellent feedback were the improvement in knowledge on the complications of CVC insertion, and the valuable hands on simulated sessions. Structured lectures were able to impart knowledge on potential complications and strategies related to CVC insertion, and how to prevent these complications. This result was obtained from a survey after the training was completed for the residents involved, and remained consistent three to four months after the training (annex 1, table 1).

There was also an increase in mean confidence rating for insertion of the CVC, from 5.26 to 6.1 ( $0=$ no confidence; $10=$ very confident) (annex 1, table 2).

A survey of CVC PLUS showed that $13 / 15(86.7 \%)$ of the procedures found it easy to use (annex 1 , table 3 ).

Seventy three percent (11/15) found the guidewire reminder stickers on the drape useful (annex 1, table 4).

During the quality improvement measure implementation, the structured training program, CVC PLUS, and reminder stickers on drapes were successfully integrated into the CVC training program for residents in the MICU and MICA. Following the success of our quality improvement initiative these measures have been ongoing continuously, and have remained sustainable since then.

CVC PLUS was found to be cheaper compared to the conventional hospital assembled equipment. The unit cost of inserting the CVC with a conventional hospital assembled toilet and suture set with reusable cloth drapes was $\$ \$ 48.86$; the same procedure using CVC PLUS with drape cost only $\mathbf{\$} \$ 19.60$. The cost reduction was S\$29.26 per CVC insertion. The two biggest contributors to the unit price were material cost and manpower cost respectively (annex 1, table 5).

\section{Lessons and limitations}

A structured educational CVC insertion training program, integrated with a modified CVC dressing kit with drape that has reminder stickers, can reduce guidewire retention during CVC insertion with the additional benefit of producing cost savings.

One of the problems encountered early on in the implementation of the intervention was the difficulty in getting residents and nurses to use the new CVC drape. We overcame this problem by creating an instructional video.

The study was limited by a small sample size of two groups of internal medicine residents $(n=39)$ between the short period of June 2012 to February 2013. We were unable to collect data on the prior 
experiences of residents in observing or inserting a central venous catheter.

With the purpose of reducing costs and improving human manpower utilization, prepacked custom made kits have been used for various medical and surgical procedures. Prepackaged, all inclusive kits for novices have been demonstrated to be able to improve the procedure quality and save staff time resources in a controlled simulation environment.[13] Device modification can be a potential strategy in procedures to improve patient safety. Our quality improvement project showed that device modification improved cost savings.

Although we are unable to separately quantify the exact cost benefit of a modified CVC dressing kit from the simultaneous improvements resulting from education and training, the users of the modified kit provided positive feedback of its benefits. The positive feedback was in terms of the user friendliness of the kit, as well as improvement in mean confidence with regards to CVC insertion.

Prepacked, custom made kits like the CVC PLUS are cost effective in reducing material and manpower cost in medical procedures. Savings may be more significant if the prepacked custom made kits were used more frequently.

An average of 40 CVCs per month are inserted in the MICU and MICA. Adopting CVC PLUS in the MICU and MICA would translate to potential savings of $\mathbf{S} \$ 14044.80$ per year. Besides the MICU and MICA, there are four other intensive care units in our hospital (CCU,SICU, CTSICU, NICU) and two other high dependency units. An average of $188 \mathrm{CVCs}$ per month are inserted in the nonmedical ICUs and HD units, based on 2013 figures from SGH clinical governance. If these additional CVCs inserted in the other intensive care units and high dependency units were included, the potential saving increases to $\$ 80055.36$ per year.

\section{Conclusion}

Although unintentionally retained CVC guidewires are rare events, repeated case reports on retained guidewires are evidence that this event still occurs, resulting in harm and additional cost to the patient. This quality improvement project shows that a structured educational training program, and custom CVC set with a modified drape with reminder stickers are cost effective methods to eliminate guidewire retention during ultrasound guided CVC insertion.

\section{References}

1. McGee DC, Gould MK. Preventing complications of central venous catheterization. N Engl J Med 2003;348(12):1123-33.

2. Kusminsky RE. Complications of central venous catheterization. J Am Coll Surg 2007;204(4):681-96.

3. Dhanani J, Senthuran S, Olivotto R, Boots RJ, Lipman J. The entrapped central venous catheter. $\mathrm{Br} \mathrm{J}$ Anaesth 2007;98(1):89-92.
4. Eisen LA, Narasimhan M, Berger JS, Mayo PH, Rosen MJ, Schneider RF. Mechanical complications of central venous catheters. J Intensive Care Med 2006;21(1):40-6.

5. Phy MP, Neilson RW Jr. Guidewire complication with central line placement. Hosp Physician 2004;40(6):41-43, 50. http://www.turnerwhite.com/memberfile.php?PubCode $=$ hp jun04_line.pdf

6. Vannucci A, Jeffcoat A, Ifune C, Salinas C, Duncan JR, Wall M. Special article: retained guidewires after intraoperative placement of central venous catheters. Anesth Analg 2013;117(1):102-8.

7. Song $Y$, Messerlian AK, Matevosian R. A potentially hazardous complication during central venous catheterization: lost guidewire retained in the patient. J Clin Anesth 2012;24(3):221-6.

8. Gunduz Y, Vatan MB, Osken A, Cakar MA. A delayed diagnosis of a retained guidewire during central venous catheterisation: a case report and review of the literature. BMJ Case Reports 2012; doi:10.1136/bcr-2012-007064

9. Ghatak T, Azim A, Baronia AK, Ghatak NK. Accidental guide-wire loss during central venous catheterization: a report of two life-threatening cases. Indian J Crit Care Med 2013;17(1):53-4.

10. Khasawneh FA, Smalligan RD. Guidewire-Related Complications during Central Venous Catheter Placement: A Case Report and Review of the Literature. Case Rep Crit Care 2011;2011:287261.

11. Schummer W, Schummer C, Gaser E, Bartunek R. Loss of the guide wire: mishap or blunder? $\mathrm{Br} \mathrm{J}$ Anaesth 2002;88(1):144-6.

12. Pérez-Díez D, Salgado-Fernández J, Vázquez-González N, et al. Images in cardiovascular medicine. Percutaneous retrieval of a lost guidewire that caused cardiac tamponade. Circulation 2007;115(24):e629-31.

13. Fenik $Y$, Celebi N, Wagner R, et al. Prepackaged central line kits reduce procedural mistakes during central line insertion:a randomized controlled prospective trial. BMC Med Educ 2013;13:60.

\section{Declaration of interests}

Nothing to declare.

\section{Acknowledgements}

Senior Nurse Manager Soobramanee (Div of Nursing, Singapore General Hospital), Asst Director Lee Lai Chee (Div of Nursing, Singapore General Hospital), Singapore General Hospital CSSD (central sterile services department), Dr Tan Yu Yang, Dr Andrew Ong Ming Liang, Dr Nagavalli,Dr Suresh Rama Chandran, Dr Tan Aik Hau.

\section{Ethical approval}

The project is a quality improvement project and was therefore exempt from SingHealth centralised institutional review board 
BMJ Quality Improvement Reports

(CIRB) review. 